\title{
The Automatic Strike-Change Phenomenon: When Running Barefoot Patients Change from a Heel Strike to a Forefoot Strike Without Any Instruction
}

\author{
Wes Zimmermann ${ }^{1,2, ~}$, Naomi van Valderen ${ }^{1}$ \\ ${ }^{1}$ Department of Military Sports Medicine, Royal Netherlands Army, Utrecht, The Netherlands \\ ${ }^{2}$ Department of Military and Emergency Medicine, Uniformed Services University of the Health Sciences, Bethesda, MD, USA
}

Email address:

wesselzimmermann@hotmail.com(W.Zimmermann),wo.zimmermann@mindef.nl(W. Zimmermann)

${ }^{*}$ Corresponding author

\section{To cite this article:}

Wes Zimmermann, Naomi van Valderen. The Automatic Strike-Change Phenomenon: When Running Barefoot Patients Change from a Heel Strike to a Forefoot Strike Without Any Instruction. International Journal of Sports Science and Physical Education.

Vol. 6, No. 1, 2021, pp. 1-7. doi: 10.11648/j.ijsspe.20210601.11

Received: February 17, 2021; Accepted: March 2, 2021; Published: March 10, 2021

\begin{abstract}
The department of Military Sports Medicine of the Royal Netherlands Army has an ongoing research line 'Exercise-Related Leg Pain' since 2011. This study investigated which immediate changes occur in running technique while running barefoot or in minimalist running shoes on a treadmill, which proportion of service members automatically changed from a heel strike landing to a forefoot strike landing without any instruction to do so, and whether these patients experienced a subsequent gait retraining program as easier and completed it faster or with a better clinical outcome. In total, data from 53 patients with exercise-related leg pain were used to answer the research questions. Running barefoot, $24.5 \%$ of patients automatically changed from a heel strike landing to a forefoot strike landing ( $16.7 \%$ of the men, $54.5 \%$ of the women), in minimalist shoes the percentage of automatic strike-changers was $5.7 \%$ (2,4\% of men, $16.2 \%$ of women). Running barefoot, the patients used shorter strides and a higher cadence than in traditional running shoes and the vertical ground reaction forces under the midfoot and heel were lower. Automatic strike-changers were not better candidates for the treatment program, in terms of subjective ease of the gait retraining sessions and duration or outcome of the treatment program. Running barefoot is a helpful methodology to facilitate gait retraining.
\end{abstract}

Keywords: Exercise-related Leg Pain, Gait Retraining, Landing Patterns, Barefoot Running, Military

\section{Introduction}

Gait retraining as a therapy for overuse injuries of the lower extremity has received increasing attention in recent years. [1] In the sports medical context, gait retraining refers to a training / rehabilitation program in which a permanent change in running technique is the goal. Diagnoses in which changing running technique may contribute to a reduction of reinjury rates include PatelloFemoral Pain Syndrome (PFPS), Iliotibial Band Friction Syndrome (ITBS), Medial Tibial Stress Syndrome (MTSS), Chronic Exertional Compartment Syndrome (CECS), Achilles Tendinopathy and Plantar Fasciopathy (PF). [1] These overuse injuries are clearly related to running and marching and are commonly seen in the military. For example, it has been established that lower leg symptoms (MTSS and CECS) are in the top three of injuries that lead to dropout in basic military training. [2] Overuse injuries of the knee, such as PFPS and ITBS, have been consistently in the top three of overuse injuries in the Netherlands Army until most recently. [3] This article reports on a study of the "automatic strike-change" phenomenon, which occurs when running barefoot and in minimalist running shoes, in some patients with lower leg overuse injuries who receive gait retraining. [2]

\subsection{Evidence for Gait Retraining as Therapy}

The exact pathophysiological mechanism of most lower extremity overuse injuries is unknown. [2] In recent years, much information has been gathered about risk factors for the development of running injuries. The relationship between the 
impact of landing on the surface (vertical ground reaction forces) and the occurrence of these overuse injuries is now regarded as convincing. [4] In addition, it is well known that overuse injuries of the lower extremity often reoccur upon resumption of the previous training load. [2] The question is to what extent an unfavorable running technique is the sustaining factor responsible for the recurrence of these injuries? [1, 4] The goal of gait retraining as therapy is to reduce the vertical ground reaction forces that the runner encounters per step (for example in the case of MTSS and PF), or to reduce the work of the muscles that are overloaded, such as the m. tibialis anterior in CECS. [5]

From 2011 onward, the department of sports medicine of the Royal Netherlands Army has had an ongoing research line "Exercise-related Leg Pain". Most studies by the department have been conducted on young service members with overuse injuries of the lower legs. Gait retraining as part of a comprehensive treatment protocol was introduced more than 10 years ago and criteria for application of interventions have been established (see Table 1).

Table 1. Standard care for exercise-related leg pain: criteria for application of an intervention.

\begin{tabular}{|c|c|}
\hline Intervention & Criterion \\
\hline Stretching & $\begin{array}{l}\text { Gastrocnemius tightness = minimal angle compared to a vertical line: } 70 \text { degrees or more; } \\
\text { Soleus tightness = maximal distance of the big toe } 5 \mathrm{~cm} \text { from the wall or less. }\end{array}$ \\
\hline Strengthening & Calve strength insufficient: not able to perform 30 consecutive calve raises on one leg. \\
\hline Massage hypertonic m. plantaris & m. plantaris palpation painful (patient in prone position). \\
\hline Dry needling of trigger points & Medial and lateral gastrocnemius: if patient identifies the calve as a pain location. \\
\hline Compression stockings / sleeves & Not given to patients with proven anterior CECS (ICPM $\geq 35 \mathrm{~mm} \mathrm{Hg}$ ). \\
\hline $\begin{array}{l}\text { Extracorporeal Shockwave Therapy } \\
\text { (ESWT) }\end{array}$ & $\begin{array}{l}\text { For MTSS only: once a week, } 4 \text { sessions; each session } 2000 \text { radial shocks, frequency } 8 \text { per second and intensity } \\
2.5 \text { bar, on the medial tibial border. }\end{array}$ \\
\hline Vitamin D supplementation & If MTSS is present: criterion: $<50 \mathrm{nmol} / 1$ means insufficient, supplementation required; optimal $75 \mathrm{nmol} / 1$. \\
\hline New running shoes & $\begin{array}{l}\text { If patient describes a relation between symptoms and shoes; Every year or } 500 \text { miles }(800 \mathrm{~km}) \\
\text { Minimalist shoes are discouraged. }\end{array}$ \\
\hline Customized anti-pronation inlays & $\begin{array}{l}\text { If navicular drop is positive }(>0.5 \mathrm{~cm}) \text { and if over-pronation is established with slow motion video analysis of } \\
\text { barefoot running. }\end{array}$ \\
\hline $\begin{array}{l}\text { Maintaining fitness with low impact } \\
\text { training }\end{array}$ & $\begin{array}{l}\text { Resume three moments of low impact exercise per week. Keep leg pain scores } \leq 3 \text { (on a Numeric Pain Rating } \\
\text { Scale } 0-10 \text { ). }\end{array}$ \\
\hline $\begin{array}{l}\text { Gait retraining while running in sports } \\
\text { shoes and boots }\end{array}$ & $\begin{array}{l}\text { Four cues for running: } \\
\text { Change to a ball-of-foot strike (reduce heel strike), when applicable. } \\
10 \% \text { reduction of stride length; } \\
\text { Strive for cadence } 180 / \text { minute; } \\
\text { Increase knee lift } 1-2 \mathrm{~cm} \text {. }\end{array}$ \\
\hline Gait retraining while marching in boots & $\begin{array}{l}\text { Two cues for marching: } \\
5 \% \text { increase in cadence from preferred; } \\
\text { Reduce force and dorsal flexion angle of heel strike. }\end{array}$ \\
\hline Progressive running schedule & $\begin{array}{l}\text { Week 1-6: run twice a week, end goal = a } 15 \text { minute uninterrupted run, pain free with new running technique; } \\
\text { Week 7-12: run twice or three times per week, end goal = a } 30 \text { minute uninterrupted run, pain free, with new } \\
\text { running technique. }\end{array}$ \\
\hline
\end{tabular}

CECS $=$ Chronic Exertional Compartment Syndrome

MTSS $=$ Medial Tibial Stress Syndrome

ICPM = intracompartmental pressure measurement

$\mathrm{Mm} \mathrm{Hg}=$ millimeter Mercury

Several years of clinical experience led to the observation that a lasting change in running technique can be achieved with approximately five individual 20-minute training sessions supplemented with home training assignments spread over a period of four to five months. [5] Three cues to change running technique were used consistently and studied:

1. switch to a forefoot strike;

2. increase cadence (at the same running speed, target value 180 steps/minute);

3. keep the upper body upright (do not lean forward or backward).

The combination of these three cues resulted in a 70 percent reduction in vertical ground reaction forces on the heel. Despite the change to forefoot striking, the maximum ground reaction forces under the forefoot were also reduced by about $15 \%$. [5] Using the same three coaching cues, the new running technique was also found to be well applicable when running in military boots. [6] Service members with exercise-related leg pain (MTSS, CECS or a combination of these) have indicated that they do not find learning the new running technique difficult and that they believe that the new running technique has contributed to their recovery. [7] Finally, a study found that adding gait retraining to the conservative treatment program for soldiers with CECS has led to a large decrease (about 80\%) in the demand for surgical treatments for CECS in a national Military Hospital. [8] In summary, it can be said that in recent years, especially in military health care, a convincing basis has been laid for gait retraining as a therapy for overuse injuries of the lower leg. [2, 5-9]

\subsection{Research Questions Arising from Clinical Practice}

During the execution of the treatment programs with gait 
retraining, the physicians and therapists gain valuable clinical experience from which new research questions arise. For example, it is a common part of the gait retraining program to ask patients to run barefoot on the treadmill for a short time. The purpose of this is to assess whether patients overpronate when running barefoot and for that reason should be provided with custom made insoles. A recurring observation was that some service members, when running barefoot on the treadmill, without instruction, automatically changed from a heel strike landing (Figure 1) to a forefoot strike landing (Figure 2) and shorter stride length; unknowingly performing two of the three essential gait retraining cues. A limited search in the literature made it clear that this phenomenon had already been described and can also occur to a lesser extent when running on so-called minimalist running shoes. [10] The characteristic properties of minimalist running shoes are: low weight, high flexibility, no to little heel height, no to little cushioning and no built-in corrective elements (figure 3). [11] This change in gait pattern when running barefoot raised several research questions. With the ultimate aim of optimizing gait retraining for service members with lower leg overuse injuries, the following four research questions were formulated about running barefoot and in minimalist running shoes:

1. What are the immediate changes in stride length, cadence, and vertical ground reaction forces when running on a treadmill, barefoot or in minimalist running shoes?

2. What percentage of soldiers with lower leg complaints automatically (without instruction) change from a heel strike landing to a forefoot strike landing when they run on a treadmill barefoot or in minimalist running shoes?

3. Do the patients who automatically change from heel to forefoot striking while running on a treadmill barefoot experience less difficulty in a gait retraining program emphasizing forefoot strike and smaller strides?

4. Do the patients who automatically change from heel to forefoot striking while running on a treadmill barefoot complete the treatment program faster or with a better clinical outcome?

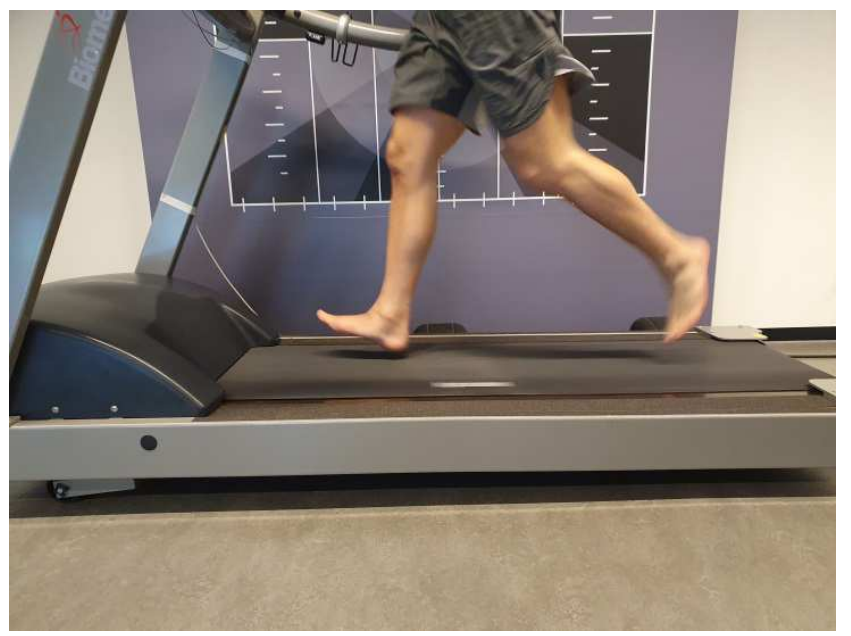

Figure 1. A heel striker, when running barefoot.

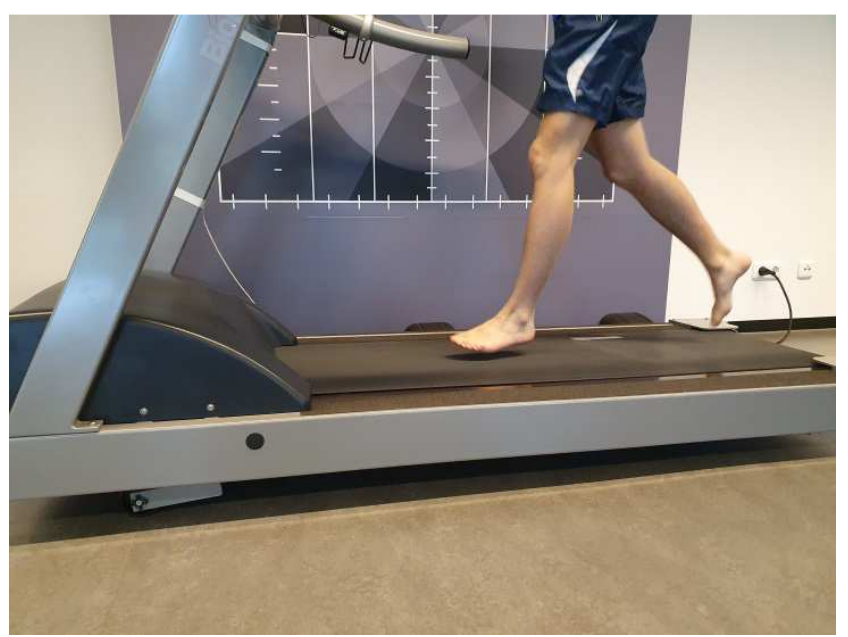

Figure 2. A forefoot striker, when running barefoot.

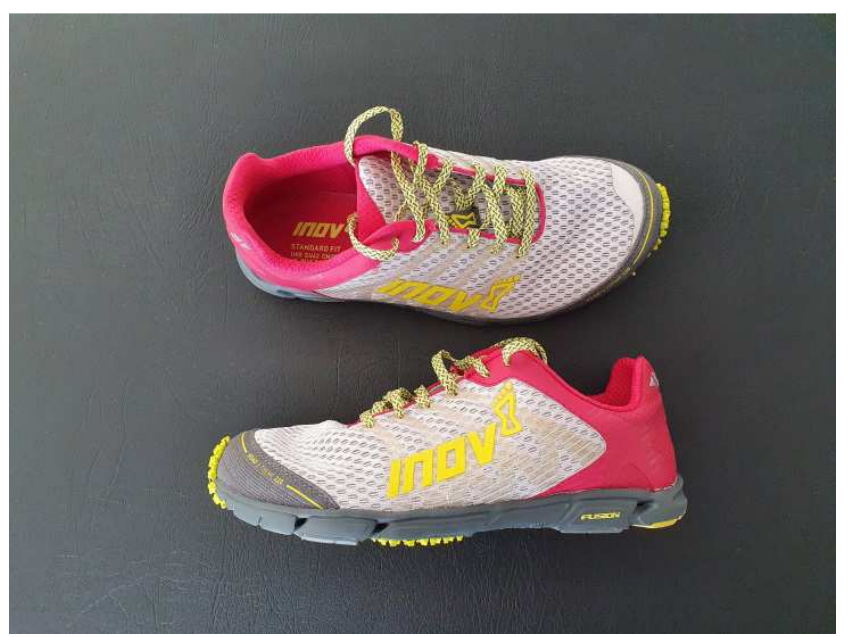

Figure 3. The minimalist running shoes used in this study.

\section{Methods}

This study includes clinical data of 53 soldiers (42 men, 11 women) with exercise-related leg pain who started an outpatient treatment program between September 2017 and April 2018. The formulated research questions could be answered with data collected during regular care.

Characteristics of the military patients included in this study were taken from the medical files: height $(\mathrm{m})$, weight $(\mathrm{kg})$, body mass index (BMI), duration of symptoms (months), recurrence of symptoms (yes / no), for an overview see table 2.

No permission is required from a medical ethics committee to present data from the treatment results from our own department. However, all patients have given written permission for the collection and anonymous processing of data from their medical file.

The most important inclusion criterion was: the patient had to be classified as a "heel striker" when running on his own, traditional running shoes at the time of intake for treatment. A heel striker was defined as a service member who, on slow motion video analysis of running technique, had a visual heel first landing and a vertical ground reaction force on the heel $>$ 400 Newton $(\mathrm{N})$. The same criteria were used when running 
barefoot and in minimalist shoes.

Table 2. Characteristics of the military patients included in this study (average, standard deviation or percentage).

\begin{tabular}{lllll}
\hline & Men $(\mathbf{n}=\mathbf{4 2})$ & SD $/$ percentage & Women $(\mathbf{n}=\mathbf{1 1})$ & SD $/$ percentage \\
\hline age (years) & 23.2 & 3.0 & 23.6 & 2.9 \\
height $(\mathrm{m})$ & 1.79 & 0.07 & 1.67 & 0.05 \\
weight $(\mathrm{kg})$ & 83.4 & 11.2 & 73.2 & 9.0 \\
BMI & 26.0 & 3.4 & 26.4 & 3.6 \\
Duration of symptoms (months) & 13.9 & 13.4 & 8.4 & 3.7 \\
Re-injury (yes) & 18 & $42.8 \%$ & 3 & $27.3 \%$ \\
\hline
\end{tabular}

$\mathrm{m}=$ meter, $\mathrm{kg}=$ kilogram, BMI $=$ Body Mass Index.

Exclusion criteria were: 1. Age> 30 years; 2. Previous gait retraining; 3. Fasciotomy less than 1 year ago; 4. Psychological complaints or other conditions that could influence gait retraining; 5. Relevant data missing from the medical file.

Research questions 1 and 2 could be answered because all military patients ran three times, in three different running shoe conditions, for 30 seconds on an instrumented treadmill (H / P / Cosmos Mercury, Nussdorf-Traustein, Germany) (speed $10 \mathrm{~km} / \mathrm{h}$, incline 1 percent) at the beginning of the gait retraining program. The treadmill can, in collaboration with software (Zebris), quickly produce biomechanical measurements such as stride length, cadence (step frequency) and vertical ground reaction forces under three sections of the foot (forefoot, midfoot and heel). High speed video cameras were placed to the side and behind the treadmill. These were used in assessing the striking technique while running. (Figure 4).

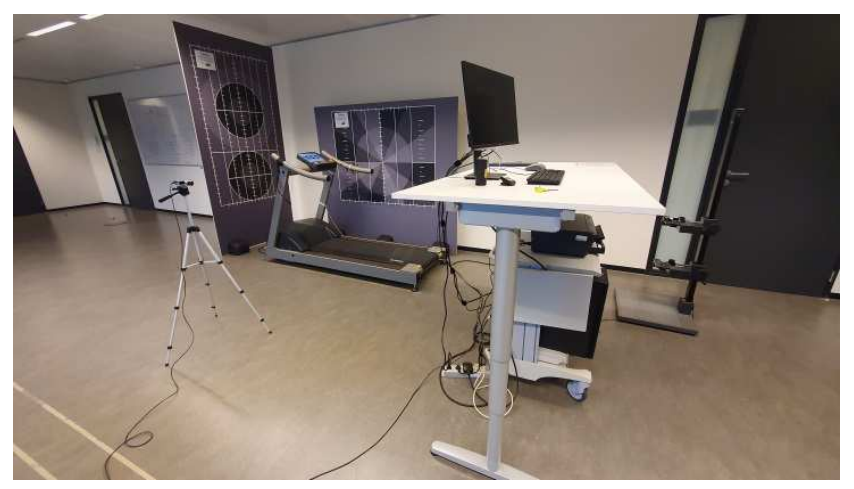

Figure 4. High speed video cameras and the treadmill used in this study.

Three running shoe conditions were measured, always in the same order: 1 . own (traditional) running shoes; 2 . barefoot; 3. minimalist running shoes (from the Inov8 brand). It was recorded when the patient executed an automatic strike-change. This was defined as a soldier who is a heel striker in his own (traditional) running shoes and not a heel striker when running barefoot or in minimalist running shoes, within 30 seconds and without any instruction to adjust running technique (see the definition of a heel striker previously described).

All enrolled military patients completed the outpatient treatment program for exercise-related leg pain as described in previous publications from our department (see Table 1). [5] Research question 3 (do automatic strike-changers find it easier to learn the new running technique?) could be answered with one multiple-choice question to all participants, at the end of the treatment program. This question had seven alternatives to score the perceived difficulty of learning and using the new running technique. A score of 1 meant "very difficult" and a score of 7 meant "very easy".

Research question 4 (do the patients who change strike automatically complete the treatment program faster and with a better clinical outcome?) could be answered by taking treatment duration in number of days and the SANE scores at the start and end of the treatment program from the medical file. The Single Assessment Numerical Evaluation (SANE) score is a patient-reported subjective score developed in the military health care system. It concerns one question: "how do you rate your lower leg today as a percentage of normal, on a 0-100 scale, with 100 being normal". [12]

Differences in measured values were tested with a paired t-test or an unpaired t-test, where appropriate. Significance was set at $p<0.05$. All statistical tests were performed with SPSS version 27.0.

\section{Results}

\subsection{Research Question 1}

Tables $3 \mathrm{~A}$ and $3 \mathrm{~B}$ show the measurement values of the three running shoe conditions at the start of the treatment program. Running barefoot or in minimalist shoes immediately, without instruction, leads to variations in stride length, cadence and the maximal vertical ground reaction forces under sections of the foot. The measurement values for running barefoot deviate significantly more often from the measurements in traditional running shoes than is the case for the measurements in minimalist running shoes.

\subsection{Research Question 2}

When running barefoot, $24.5 \%$ of patients automatically change from a heel strike landing to a forefoot strike landing without any instruction ( $16.7 \%$ of men, $54.5 \%$ of women). When running in minimalist running shoes, the percentage of automatic strike-changers is $5.7 \%(2.4 \%$ of men, $16.2 \%$ of women).

\subsection{Research Question 3 and 4}

Table 4 shows the difference in treatment duration (136 versus 163 days), the value of the SANE scores (increase 28.8 versus 21.9 ) and the perceived difficulty of changing running 
technique (3.6 versus 3.8 on a scale of 5) of patients who continue to heel strike when running barefoot (non- automatic strike-changers), versus the patients who do change from a forefoot strike to a heel strike without instruction (automatic strike-changers). The values found are not significantly different for the two patient groups.

Table 3. A and B. Measured values at intake, during 30 seconds of running at $10 \mathrm{~km} / \mathrm{h}$ and 1 percent incline, three shoe conditions, without any instruction. 3 A: men, $3 B:$ women. * $p<0.05$; the measured values are significantly different from those in traditional running shoes. \# $p<0.05 ;$ the measured values are significantly different from the measurement in minimalist running shoes.

\begin{tabular}{|c|c|c|c|c|c|c|}
\hline $\mathbf{3 A}$ & Running shoes & $95 \% \mathrm{CI}$ & Barefoot & $95 \% \mathrm{CI}$ & Minimalist shoes & $95 \% \mathrm{CI}$ \\
\hline $\operatorname{Men}(n=42)$ & average & & average & & average & \\
\hline Stride length $(\mathrm{cm})$ & 207 & $203 ; 210$ & $201^{*}, \#$ & $198 ; 205$ & 206 & $202 ; 209$ \\
\hline Cadence (steps/min) & 160 & $158 ; 163$ & $165^{*}$, \# & $162 ; 168$ & 162 & $159 ; 164$ \\
\hline F-forefoot max $(\mathrm{N})$ & 1083.0 & $1021.9 ; 1144.0$ & $1060.8^{*}$ & $1009.9 ; 1111.7$ & 1096.1 & $1035.0 ; 1157.2$ \\
\hline F-midfoot $\max (\mathrm{N})$ & 644.4 & $600.9 ; 688.5$ & $354.8^{*}$,\# & $314.7 ; 395.0$ & $534.3^{*}$ & $490.1 ; 578.5$ \\
\hline F-heel $\max (\mathrm{N})$ & 661.8 & $633.5 ; 690.1$ & $617.4^{\#}$ & $569.0 ; 665.9$ & 744.1 & $709.3 ; 779.0$ \\
\hline Automatic strike-change & n.a. & & $16.7 \%$ & & $2.4 \%$ & \\
\hline 3B & Running shoes & $95 \% \mathrm{CI}$ & Barefoot & $95 \% \mathrm{CI}$ & Minimalist shoes & $95 \% \mathrm{CI}$ \\
\hline Women $(\mathrm{n}=11)$ & average & & average & & average & \\
\hline Stride length $(\mathrm{cm})$ & 201 & $193 ; 208$ & $194^{*}$,\# & $187 ; 201$ & $197^{*}$ & $190 ; 203$ \\
\hline Cadence (steps/min) & 166 & $159 ; 172$ & $171^{*}$,\# & $165 ; 177$ & 169 & $164 ; 174$ \\
\hline F-forefoot max $(\mathrm{N})$ & 982.8 & $864.9 ; 1100.6$ & 953.9 & $891.5 ; 1016.2$ & 1000.4 & $858.6 ; 1142.3$ \\
\hline F-midfoot max $(\mathrm{N})$ & 465.2 & $387.3 ; 543.1$ & $326.5^{*}, \#$ & $251.2 ; 401.9$ & $424.0^{*}$ & $338.1 ; 510.0$ \\
\hline Automatic strike- change & n.a. & & $54.4 \%$ & & $16.2 \%$ & \\
\hline
\end{tabular}

$n=$ number, $\mathrm{cm}=$ centimeter, $\min =$ minute, $F=$ force $=$ maximum vertical ground reaction forces, per section of the foot, $N=$ Newton, $95 \%$ CI $=95 \%$ confidence interval, n.a. $=$ not applicable

Table 4. Evaluation of the treatment program for exercise-related leg pain for non-automatic strike-changers vs automatic strike-changers (means, standard deviation).

\begin{tabular}{|c|c|c|c|c|}
\hline & Non-automatic strike-changers (barefoot) & SD / \% & Automatic strike-changers (barefoot) & SD / \% \\
\hline Number of patients & 40 & $75 \%$ & 13 & $25 \%$ \\
\hline Treatment duration (days) & 136 & 63 & 163 & 47 \\
\hline Number of gait retraining sessions & 5.0 & 0.9 & 4.9 & 1.6 \\
\hline SANE in & 49.4 & 15.9 & 50.4 & 15.2 \\
\hline SANE out & 78.2 & 15.4 & 72.3 & 18.1 \\
\hline SANE increase & 28.8 & $58.2 \%$ & 21.9 & $43.5 \%$ \\
\hline
\end{tabular}

SANE = Single Assessment Numerical Evaluation, SANE in = score at intake, SANE out = score at end of treatment program.

\section{Discussion}

This study was inspired by clinical questions of military sports medicine physicians and therapists while providing gait retraining as therapy. The focus was on the "automatic strike-change" phenomenon, changing from a heel strike landing to a forefoot strike landing when running barefoot, without instruction to do so. Findings in this study are: 1 . When barefoot, service members run with smaller steps and higher cadence than in traditional sports shoes. When barefoot, $16.7 \%$ of male patients automatically change from a heel strike to a forefoot strike without any instruction, in female patients $54.5 \%$ change strike. When running in minimalist running shoes, the percentage of strike-changers is $2.4 \%$ for men and $18.2 \%$ for women. It was also investigated whether service members who demonstrate the strike-change phenomenon experience less difficulty learning the new running technique and complete the treatment program faster or with a better clinical outcome. The answer to these research questions is that this is not the case.

\subsection{Shoes Offer Little Reduction of Ground Reaction Forces}

The biomechanical measurements presented in Tables 3A and $3 \mathrm{~B}$ are consistent with previous findings for healthy runners. [10, 13, 14] In summary it can be concluded that when running barefoot subjects perform smaller steps and a higher cadence. [13, 14] Contrary to expectations, running shoes do not provide much protection (cushioning) against the maximal vertical ground reaction forces, when compared to the barefoot condition. [8] The biomechanical measurements for running in minimalist running shoes are more like those of running in regular running shoes than those of running barefoot. $[13,14]$ It was therefore previously concluded that running in minimalist running shoes is not the same as running barefoot. [13]

\subsection{Changing Strike Patterns}

The change in foot placement (degree of heel landing) when 
running barefoot has also been observed and measured before. $[10,14]$ In these studies, the so-called Strike Index was used. This is the measured location of the center of pressure in relation to the foot length, at the time of the first foot contact with the ground. A foot placement at the very back of the heel corresponds to a Strike Index 0 (zero percent of the foot length), a foot placement at the very front of the toes corresponds to a Strike Index 100 (100\% of the foot length). As an indication, in 14 recreational runners the Strike Index shifted from 18.6 on traditional running shoes to 27.0 in bare feet, indicative of a displacement of the first foot contact point of the heel towards the forefoot. [14] However, these were average values. It was not indicated how many subjects changed completely to a forefoot landing, defined as a Strike Index greater than 68. In our study, the Strike Index is not reported, but rather the percentage of patients changing from a heel strike to a forefoot strike, hoping to answer a clinically relevant question: Are automatic strike-changers better candidates for a therapeutic gait retraining program? This turned out not to be the case.

\subsection{Gender Differences}

This study was not designed to focus on a difference between male and female patients. However, it is noticeable that a higher proportion of women change to a forefoot strike $(54.5 \%)$ than men $(16.7 \%)$ when running barefoot. There is also a difference in minimalist running shoes, $18.2 \%$ for women and $2.4 \%$ for men. A possible explanation is that the Pressure Pain Threshold value on the heel is lower for women than for men. [15] If women experience pain on the heel more easily, they are more likely to avoid landing on the heel when running barefoot. Here, follow-up research with a larger population of men and women is desirable. This Pressure Pain Threshold on the heel may be the explanation for the "strike-change" phenomenon.

\subsection{Study Limitations}

Several limitations of this study must be addressed. It was a medical file study. Patients were only included if the treatment program had been completed and the desired information was available in the medical file. The omission of patients who started a course of treatment, but did not complete it, should always lead to a cautious interpretation of findings. Female patients were under-represented in this study. This is very common in military studies, because the ratio of Defense employees is about $90 \%$ male to $10 \%$ female. The answer to research question 4 (duration and effectiveness of the treatment program) must also be interpreted with caution. The time of completion of the treatment program and the SANE score at completion were regularly influenced by patient availability (absence for military tasks or vacation). This means that the reported treatment duration and SANE score (out) should also be assessed with caution.

\subsection{Clinical Relevance}

It was already known from the literature that running barefoot and in minimalist running shoes are not the same. The value of this study seems mainly to substantiate the practical experience that when providing gait retraining as a therapy, running barefoot is a useful step in the methodology to teach the transfer from heel striking to forefoot striking. While barefoot, the patient will experience more clearly that heel landings produce greater vertical ground reaction forces compared to forefoot landings. The instruction "take smaller steps" is also easier to perform barefoot than in running shoes, almost all subjects already do this automatically. If during the gait retraining program the patient forgets the intended new running technique for a moment (especially the forefoot strike and the smaller steps), then running barefoot for a brief moment is often the cue that will restore the desired technique. Finally, an additional tip for practice. Gait retraining as therapy is often performed on a treadmill, because speed, incline and other conditions are easiest to control. At the end of the gait retraining program, it is important that the therapist makes sure the new running technique is transferred from the running laboratory to the natural environment. Sometimes a patient masters the new technique in the lab, but "forgets" to apply the new technique when outdoors. It would be unfortunate if this final step of the gait retraining process is forgotten.

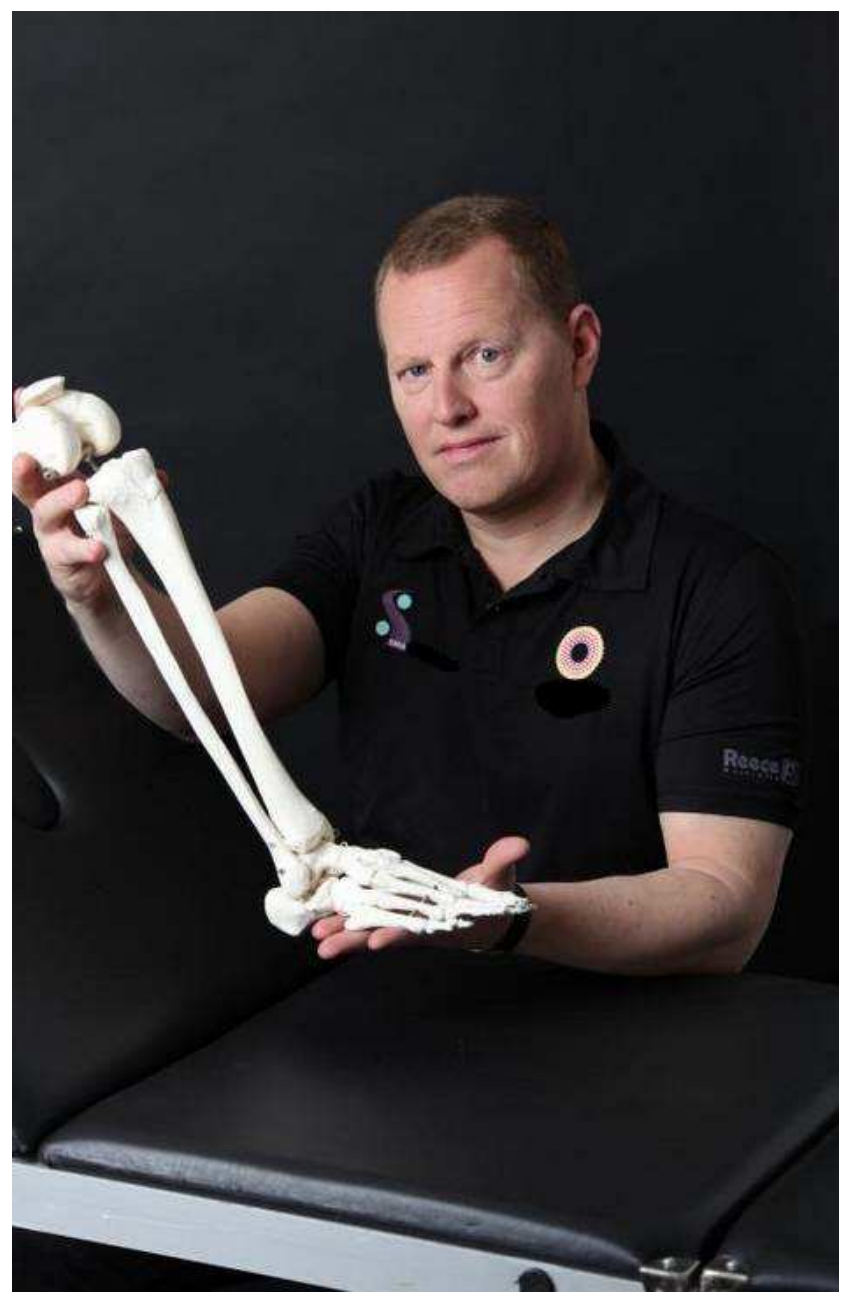

Figure 5. Wes Zimmermann, $M D, P h D$. 


\section{Conclusion}

Running in different shoe conditions leads spontaneously, without instruction, to differences in stride length, cadence and the maximal vertical ground reaction forces under sections of the foot. The measurements for barefoot running deviate more often from the measurements in traditional running shoes than the measurements in minimalist running shoes. When running barefoot, $24.5 \%$ of the patients change (automatically) without instruction from a heel strike landing to a forefoot strike landing $(16.7 \%$ of the men, $54.5 \%$ of the women). When running in minimalist running shoes, the percentage of strike-changers is $5.7 \%$ (2.4\% of men, $16.2 \%$ of women). Automatic strike-changers did not appear to be better candidates for a treatment program focusing on gait retraining as therapy, in terms of subjective experience of difficulty, duration or outcome of the treatment program.

\section{Acknowledgements}

We would like to thank all service members who have contributed to this study for their kind cooperation and staff members of the department of military sports medicine Utrecht, The Netherlands, for their contributions to the logistics of this study.

\section{References}

[1] Barton CJ, Bonnano DR, Carr J et al. Running retraining to treat lower limb injuries: a mixed-methods study of current evidence synthesized with expert opinion. Br J Sports Med 2016, 50, 513-526.

[2] Zimmermann WO, Helmhout PH, Beutler AI. Prevention and treatment of exercise related leg pain in young soldiers. A review of the literature and current practice in the Royal Netherlands Armed forces. J R Army Med Corps 2017, 163, 94-103.

[3] Dijksma I, Bekkers M, Spek B et al. Epidemiology and financial burden of musculoskeletal injuries as the leading health problem in the military. Mil Med 2019, 185, 3-4, e480-e486.

[4] Davis IS, Rice HM, Wearing SC. Why forefoot striking in minimalist shoes might positively change the course of running injuries. J Sport health Sci 2017, 6, 154-161.

[5] Zimmermann WO, Bakker EWP. Reducing vertical ground reaction forces: the relative importance of three gait retraining cues. Clin Biom 2019, 69, 16-20.

[6] Zimmermann WO, van Valderen NRI, Linschoten CW et al.
Gait retraining reduces vertical ground reaction forces in running shoes and military boots. Transl Sports Med 2019, 2, 90-97.

[7] Zimmermann WO, Linschoten CW, Beutler AI. Gait retraining as part of the treatment program for service members with exercise-related leg pain: preliminary clinical experiences and retention. S African J Sports Med 2017, 29.

[8] Ligthert E, Helmhout PH, van der Wurff $\mathrm{P}$ et al. Het onderbeenspreekuur in het Centraal Militair Hospitaal, deel 1. NMGT 2017, 70, 90-98. [Dutch].

[9] Zimmermann WO, Hutchinson MR, van den Berg RH et al. Conservative treatment of anterior chronic compartment syndrome in the military, with a mid-term follow up. BMJ Open Sport \& Exerc Med 2019, 5, e000532. Connors, J., \& Levy, J. (2020).

[10] Paquette MR, Zhang S, Baumgartner LD. Acute effects of barefoot, minimal shoes and running shoes on lower limb mechanics in rear and forefoot strikers. Footwear Science 2013, 5, 1, 9-18.

[11] Knapik JJ, Orr R, Pope R et al. Injuries and footwear (part 2). $\mathrm{J}$ of Special Operations Med 2016, 16, 1, 89-96.

[12] Williams GN, Gangel TJ, Arciero RA et al. Comparison of the single assessment evaluation method and two shoulder rating scales. Am J Sports Med 1999, 27, 2, 214-221.

[13] Bonacci J, Saunders PU, Hicks A et al. Running in a minimalist and lightweight shoe is not the same as barefoot: a biomechanical study. Br J Sports med 2013, 47, 387-392.

[14] Squadrone R, Rodano R, Hamill J et al. Acute effects of different minimalist shoes on foot strike pattern and kinematics in rearfoot strikers during running. Journal of Sports Sciences 2014, 33, 11, 1196-1204.

[15] Rios-Leon M, Ortega-Santiago R, Madeleine $\mathrm{P}$ et al. Topographical pressure pain sensitivity maps of the feet reveal bilateral pain sensitivity in patients with unilateral plantar heel pain. J of Orthop and Sports Phys Ther 2019, 49, 9, 640-646.

\section{Biography}

Wes Zimmermann, Lieutenant Colonel, MD, PhD (1964) is a primary care sports medicine physician working for the Royal Netherlands Army in the city of Utrecht, The Netherlands. He is specializing in the treatment of overuse injuries of the lower extremity. He is a fellow of the European College of Sports Medicine and holds an appointment as Adjunct Professor with the Uniformed Services University of the Health Sciences in Bethesda, Maryland, USA. $\mathrm{He}$ is a former international springboard diver and has a special interest in the medical aspects of Olympic springboard and platform diving. 